\title{
Gaps between classes of matrix monotone functions
}

\author{
Frank Hansen, Guoxing Ji and Jun Tomiyama *
}

April 16, 2002

\section{Introduction}

Almost seventy years have passed since K. Löwner [8] proposed the notion of operator monotone functions. A real, continuous function $f: I \rightarrow \mathbf{R}$ defined on an (non trivial) interval $I$ is said to be matrix monotone of order $n$ if

$$
x \leq y \quad \Rightarrow \quad f(x) \leq f(y)
$$

for any pair of self-adjoint $n \times n$ matrices $x, y$ with eigenvalues in $I$. We denote by $P_{n}(I)$ the set of such functions. A function $f: I \rightarrow \mathbf{R}$ is said to be operator monotone if it is matrix monotone of arbitrary orders. We evidently have $P_{n+1}(I) \subseteq P_{n}(I)$ for each natural number $n$, and

$$
P(I)=\bigcap_{n=1}^{\infty} P_{n}(I)
$$

is the set of operator monotone functions defined on $I$. If (1) holds for any pair of self-adjoint elements $x, y$ in a $C^{*}$-algebra $A$ with spectra contained in $I$, then we say that $f$ is $A$-monotone.

Löwner characterized the set of matrix monotone functions of order $n$ in terms of positivity of certain determinants (the so called Löwner determinants and the related Pick determinants) and proved that a function is operator monotone if and only if it allows an analytic continuation to a Pick function, that is an analytic function defined in the complex upper half plane

*The second author was partially supported by the National Natural Science Foundation of China(No.10071047) and the Excellent Young Teachers Program of MOE, P.R.C. 
with non-negative imaginary part. Dobsch [2] continued Löwner's investigation and gave an alternative characterization of matrix monotonicity which we shall use in this paper.

Forty years after Löwner's work W. Donoghue published a comprehensive book on the subject in which he refined Dobsch' necessary and sufficient condition for a function on an interval to be matrix monotone of order $n$, Chapter 7, Theorem VI and Chapter 8, Theorem V]. Donoghue then asserted [3, p. 84] that with this insight one may recognize that the classes $P_{n}(I)$ are all distinct for different values of $n$. We shall denote this as the (asserted) existence of gaps between the different classes of matrix monotone functions.

However, both Löwner's and Dobsch' conditions for matrix monotonicity of order $n$ are very hard to check even for $n=3$, and explicit examples of functions showing such gaps are given by Donoghue only for $n=1$ and $n=2$. Now another almost thirty years have passed after Donoghue's work and there are still, to our knowledge, no examples in the literature showing the gaps between $P_{n}(I)$ and $P_{n+1}(I)$ for arbitrary natural numbers $n$. The purpose of this article is to prove exactly the existence of such gaps for every $n$. We also characterize, for any natural number $n$, the $\mathrm{C}^{*}$-algebras $A$ with the property that any function $f \in P_{n}(I)$ is $A$-monotone. It is interesting to notice that this question is closely connected to the problem of matricial structure of operator algebras with respect to positive linear maps.

\section{The gap between $P_{n}(I)$ and $P_{n+1}(I)$}

For a positive integer $n$ let $g_{n}(t)$ be the polynomial defined by

$$
g_{n}(t)=t+\frac{1}{3} t^{3}+\cdots+\frac{1}{2 n-1} t^{2 n-1} .
$$

Following the notations in [3] we consider the matrix valued function associated with $g_{n}(t)$ and given by

$$
M_{n}\left(g_{n} ; t\right)=\left(\frac{g_{n}^{(i+j-1)}(t)}{(i+j-1) !}\right)_{i, j=1}^{n} .
$$

The following lemma is an application of standard arguments from the theory of moment problems for Hankel matrices.

Lemma 1 The matrix $M_{n}\left(g_{n} ; 0\right)$ is positive definite. 
Proof. We set

$$
b_{k}=\frac{1}{2} \int_{-1}^{1} t^{k} d t \quad \text { for } k=0,1,2, \ldots
$$

and calculate

$$
b_{k}= \begin{cases}(k+1)^{-1} & \text { if } k \text { is even, } \\ 0 & \text { if } k \text { is odd }\end{cases}
$$

Hence, we can write $g_{n}$ as

$$
g_{n}(t)=b_{0} t+b_{1} t^{2}+\cdots+b_{2 n-2} t^{2 n-1}
$$

and therefore obtain

$$
g_{n}^{(i+j-1)}(0)=(i+j-1) ! \cdot b_{i+j-2} \quad i, j=1, \ldots, n .
$$

Consequently

$$
M_{n}\left(g_{n} ; 0\right)=\left(b_{i+j-2}\right)_{i, j=1}^{n} .
$$

Now take a vector $c=\left(c_{1}, c_{2}, \ldots, c_{n}\right) \in \mathbf{C}^{n}$ and calculate

$$
\begin{aligned}
\left(M_{n}\left(g_{n} ; 0\right) c \mid c\right) & =\sum_{i=1}^{n} \sum_{j=1}^{n} b_{i+j-2} c_{j} \overline{c_{i}} \\
& =\frac{1}{2} \sum_{i=1}^{n} \sum_{j=1}^{n} \int_{-1}^{1} t^{i+j-2} c_{j} \overline{c_{i}} d t \\
& =\frac{1}{2} \int_{-1}^{1}\left|\sum_{i=1}^{n} c_{i} t^{i-1}\right|^{2} d t .
\end{aligned}
$$

It follows that the matrix $M_{n}\left(g_{n} ; 0\right)$ is positive semidefinite. Moreover, if $M_{n}\left(g_{n} ; 0\right) c=0$ we see that

$$
\sum_{i=1}^{n} c_{i} t^{i-1}=0 \quad \text { a.e. }
$$

Since this is a polynomial, it is identically zero on the interval $[-1,1]$. All entries of the vector $c$ are therefore zero and the matrix $M_{n}\left(g_{n} ; 0\right)$ is positive definite. QED

With this lemma we can show the existence of a gap between $P_{n}(I)$ and $P_{n+1}(I)$ for any positive integer $n$ and any nontrivial interval $I$ different from the whole real line. 
Theorem 2 For any natural number $n$ there exists a real number $\alpha_{n}>0$ and a function $g_{n}:\left[0, \alpha_{n}[\rightarrow \mathbf{R}\right.$ such that

(1) $g_{n}$ is matrix monotone of order $n$ on $\left[0, \alpha_{n}[\right.$.

(2) $g_{n}$ is not matrix monotone of order $n+1$ on $\left[0, \alpha_{n}[\right.$, nor is it matrix monotone of order $n+1$ on any subinterval.

Proof. Consider the polynomial $g_{n}(t)$ introduced in the proof of Lemma 1 . By the continuous dependence of eigenvalues of matrices as a function of their entries, there exists by Lemma 1 a positive number $\alpha_{n}$ such that the matrix function $M_{n}\left(g_{n} ; t\right)$ is positive definite for $t \in\left[0, \alpha_{n}\right.$. Since $g^{(2 n-3)}$ in addition is positive and convex on ]0, $\alpha_{n}$ [ we conclude, cf. [3, Chap. 8, Theorem V], that the function $g_{n}$ is matrix monotone of order $n$ on the interval $\left[0, \alpha_{n}[\right.$. The last principal matrix of order 3 of the matrix $M_{n+1}\left(g_{n} ; t\right)$ is given by

$$
\left(\begin{array}{ccc}
\frac{1}{2 n-3}+(n-1) t^{2} & t & \frac{1}{2 n-1} \\
t & \frac{1}{2 n-1} & 0 \\
\frac{1}{2 n-1} & 0 & 0
\end{array}\right)
$$

and this matrix has determinant $-(2 n-1)^{-3}$ regardless of the value of $t$. The matrix $M_{n+1}\left(g_{n} ; t\right)$ is thus not positive semi-definite and the function $g_{n}$ is not matrix monotone of order $n+1$ on any subinterval $J \subseteq\left[0, \alpha_{n}\right.$ [. This completes the proof. QED

Consider the concrete function $g_{n}$ defined in equation 2. A calculation shows that the largest possible value of $\alpha_{2}$ is 1 . It is exceedingly difficult to calculate the largest possible value for $n \geq 3$.

Proposition 3 Let either $I=[a, b[$ or $I=[a, \infty[$ for real numbers $a<b$ and take $\alpha>0$. Then there exists a bijection $h:[0, \alpha[\rightarrow I$ such that both $h$ and the inverse map are operator monotone. Likewise, with $J=] a, b]$ or $J=]-\infty, b]$, there exists a bijection $g:[0, \alpha] \rightarrow J$ such that both $g$ and the inverse map are operator monotone.

Proof. An affine map of the form $t \rightarrow c t+d$ with $c>0$ is operator monotone, and so is the inverse map $t \rightarrow c^{-1}(t-d)$. We may therefore assume that $\alpha=1$, $I=[0,1[$ or $I=[0, \infty[$, and $J=] 0,1]$ or $J=]-\infty, 0]$. The function

$$
h(t)=t(1+t)^{-1} \quad \text { with inverse } \quad h^{-1}(t)=t(1-t)^{-1}
$$


is a bijection of $[0, \infty[$ to $[0,1[$. Likewise is the function

$$
g(t)=(1-t)^{-1} \quad \text { with inverse } \quad h^{-1}(t)=1-t^{-1}
$$

a bijection of $] \infty, 0]$ to $] 0,1]$. The assertion follows since $h, h^{-1}, g, g^{-1}$ are all operator monotone functions, cf. [1, 6]. QED

Notice that we cannot find a bijection $h:] 0,1[\rightarrow \mathbf{R}$ such that both $h$ and $h^{-1}$ are operator monotone. An operator monotone function defined on the whole real line is necessarily affine, cf. [3]. Its range is therefore either a constant or the whole real line.

Corollary 4 Let $I=[a, b[$ or $I=[a, \infty[$ for real numbers $a<b$. For any natural number $n$ there exists a function $f_{n}: I \rightarrow \mathbf{R}$ such that

(1) $f_{n}$ is matrix monotone of order $n$ on $I$.

(2) $f_{n}$ is not matrix monotone of order $n+1$ on $I$, nor is it matrix monotone of order $n+1$ on any subinterval.

Let $I$ be any open real interval and take $t_{0} \in I$. Bendat and Sherman proved in [1, Theorem 3.2] that a function $f: I \rightarrow \mathbf{R}$ is matrix convex of order $n$, if and only if the function

$$
F(t)=\frac{f(t)-f\left(t_{0}\right)}{t-t_{0}}
$$

is matrix monotone of order $n$. Notice that $f$, for $n \geq 2$, automatically is differentiable and $F\left(t_{0}\right)=f^{\prime}\left(t_{0}\right)$. One may set $F\left(t_{0}\right)=\left(f\left(t_{0}\right)_{+}+f\left(t_{0}\right)_{-}\right) / 2$ for $n=1$. We also have $f(t)=f\left(t_{0}\right)+F(t)\left(t-t_{0}\right)$.

Corollary 5 Let $I=[a, b[$ or $I=[a, \infty[$ for real numbers $a<b$. For any natural number $n$ there exists a function $f_{n}: I \rightarrow \mathbf{R}$ such that

(1) $f_{n}$ is matrix convex of order $n$ on $I$.

(2) $f_{n}$ is not matrix convex of order $n+1$ on $I$, nor is it matrix convex of order $n+1$ on any subinterval.

The statement follows by combining Bendat and Sherman's result with Corollary 1 . 


\section{Characterization of $C^{*}$-algebras in terms of matrix monotone functions}

As we have discussed in [7], we may regard the question of monotonicity of functions as a kind of nonlinear version of the problem of matricial structure of operator algebras. Recall that a positive linear map $\tau$ from a $C^{*}$-algebra $A$ to a $C^{*}$-algebra $B$ is said to be $n$-positive if the map

$$
\tau_{n}:\left(a_{i j}\right)_{i, j=1}^{n} \rightarrow\left(\tau\left(a_{i j}\right)\right)_{i, j=1}^{n}
$$

is a positive map from $M_{n}(A)$ to $M_{n}(B)$. If $\tau$ is $n$-positive for all positive integers, then it is said to be completely positive.

Although the introduction of these notions by Stinespring [9] is of a much later date than the work of Löwner, they have turned out to be very important notions for the matricial structure of operator algebras i.e. $C^{*}$-algebras and von Neumann algebras. One may simply recognize this aspect by the recent publication 四 by Effros and Ruan. Meanwhile examples of $n$-positive maps which are not $(n+1)$-positive had been investigated, and it had been discussed for which types of $C^{*}$-algebras $A$ every $n$-positive map from or to $A$ for an another $C^{*}$-algebra $B$ is also $(n+1)$-positive. In this sense, gaps between $P_{n+1}(I)$ and $P_{n}(I)$ are nonlinear versions of the above sort of problems. We are thus naturally led to the problem of the characterization of those $C^{*}$-algebras $A$ on which every matrix monotone functions of order $n$ is $A$-monotone. The following theorem is a generalization of a previous result [7, Theorem 1] where the two last authors essentially treated the gap between $P_{1}(I)$ and $P_{2}(I)$. In this investigation we reach the same kind of $C^{*}$-algebras as in the study of positive linear maps by the third author [11].

Theorem 6 Let $A$ be a $C^{*}$-algebra, and let $I$ be an interval of the form $I=[a, b[$ or $I=[0, \infty[$ for real numbers $a<b$. The following assertions are equivalent:

(1) Every matrix monotone function of order $n$ defined on I is A-monotone.

(2) The dimension of every irreducible representation of $A$ is less or equal to $n$.

(3) Every n-positive linear map from/to $A$ for another $C^{*}$-algebra $B$ is completely positive.

Proof. (1) $\Rightarrow(2)$ : We first notice that we, without loss of generality, may choose the interval $I=[0, \infty[$. Suppose that $A$ had an irreducible representation $\pi$ on a Hilbert space $H$ whose dimension is greater than $n$. Take an 
$(n+1)$-dimensional projecton $e$ in $H$. We then have $\pi(A) e=B(H) e$ by $[10$, Theorem 4.18], hence

$$
e \pi(A) e=e B(H) e=B(e H) \cong M_{n+1} .
$$

Let $B$ be the $C^{*}$-subalgebra of $A$ defined by setting

$$
B=\{a \in A \mid \pi(a) e=e \pi(a)\}
$$

By the theorem cited above, the restriction of $\pi$ to $B$ is a $*$-homomorphism onto $e B(H) e$. We choose a function $f$ in $P_{n}(I)$ which is not matrix monotone of order $n+1$, cf. Corollary 4 . Let $c$ and $d$ be arbitrary positive elements in $e B(H) e$ with $c \leq d$. It is easily verified that we can find positive elements $a$ and $b$ in $B$ such that $a \leq b, \pi(a)=c$ and $\pi(b)=d$. Since $a \leq b$ we obtain $f(a) \leq f(b)$ by the assumption, whence

$$
f(c)=f(\pi(a))=\pi(f(a)) \leq \pi(f(b))=f(\pi(b))=f(d) .
$$

Therefore, $f$ is matrix monotone of order $n+1$ on $I$, a contradiction.

$(2) \Rightarrow(1)$ : Take a function $f$ in $P_{n}(I)$ and let $a$ and $b$ be self-adjoint elements in $A$ with spectra contained in $I$ such that $a \leq b$. We consider an irreducible representation $\pi$ of $A$. Since also the spectra of $\pi(a)$ and $\pi(b)$ are contained in $I$, we obtain by the assumptions that

$$
\pi(f(a))=f(\pi(a)) \leq f(\pi(b))=\pi(f(a)) .
$$

It follows that $f(a) \leq f(b)$, thus $f$ is $A$-monotone.

$(2) \Leftrightarrow(3)$ : The assertion is proved in [1]. QED

\section{References}

[1] J. Bendat and S. Sherman, Monotone and convex operator functions, Trans. Amer. Math. Soc 79 (1955), 58-71.

[2] O. Dobsch, Matrixfunktionen beschränkter Schwankung, Math. Z. 43 (1937), 353-388.

[3] W. Donoghue, Monotone Matrix Functions and Analytic Continuation, Springer 1974.

[4] E.G. Effros and Z.-J. Ruan, Operator spaces, London Math. Soc. monograph vol. 23, 2000. 
[5] F. Hansen, Operator monotone functions of several variables, Math. Inequal. Appl., (to appear).

[6] F. Hansen and G.K. Pedersen, Jensen's inequality for operators and Löwner's theorem, Math. Ann. 258 (1982), 229-241.

[7] G. Ji and J. Tomiyama, On characterizations of commutativity of $C^{*}$ algebras, preprint.

[8] K. Löwner, $\ddot{U}$ ber monotone Matrixfunktionen, Math. Zeit. 38 (1934), 177-216.

[9] W.F. Stinespring, Positive functions on $C^{*}$-algebras, Proc. Amer. Math. Soc. 6 (1955), 211-216.

[10] M. Takesaki, Theory of operator algebras I, Springer 1979.

[11] J. Tomiyama, On the differnce of $n$-positivity and complete positivity in $C^{*}$-algebras, J. Funct. Anal. 49 (1982), 1-9.

[12] J. Tomiyama, On the geometry of positive maps in matrix algebras II, Linear Algebra Appl. 69 (1985), 169-177.

Frank Hansen (frank.hansen@econ.ku.dk)

Institute of Economics, Copenhagen University, Denmark.

Guoxing Ji (gxji@snnu.edu.cn)

College of Mathematics and Information Science, Shaanxi Normal University, Xi'an, 710062, P.R. China.

Jun Tomiyama (jtomiyama@fc.jwu.ac.jp)

Department of Mathematics and Physics, Japan Women's University, Mejirodai Bunkyo-ku, Tokyo, Japan. 